\title{
Bajo nivel de crecimiento de la industria textil ecuatoriana: ¿Elevada concentración industrial o problemas productivos estructurales? ${ }^{1}$
}

\author{
URL: http://revistas.uta.edu.ec/erevista/index.php/bcoyu/article/view/691 DOI: http://dx.doi.org/10.31164/bcoyu.21.2019.691 \\ Sebastián Amaluisa-Peñaranda ${ }^{2}$ \\ Fecha de recepción: 25 de marzo de 2019 \\ Fecha de aceptación: 27 de mayo de 2019
}

\begin{abstract}
Resumen
La industria textil históricamente ha sido relevante para la economía ecuatoriana debido a su influencia sobre agregados macroeconómicos como el producto interno bruto (PIB) y el empleo, presenta problemas de crecimiento reflejados en su nivel de producción. Este artículo tiene por objeto determinar la principal causa del bajo nivel de crecimiento de la industria; para lo cual, se comprobará, por un lado, la relación entre concentración industrial y el nivel de crecimiento, y por otro, la existencia de barreras de entrada y problemas productivos que presenta la industria como causa de su bajo nivel de crecimiento. Se desarrolla una investigación descriptiva de tipo cuantitativa y cualitativa, como principal resultado, se encuentra que la industria textil ecuatoriana mantiene un bajo nivel de concentración y que no se establece la existencia de fuertes barreras de entrada en la industria, por lo que no son causa directa del bajo nivel de crecimiento de la industria. Sin embargo, la industria presenta problemas estructurales derivados del propio accionar de las empresas y de la política pública, la cual es la principal causa del bajo nivel de crecimiento industrial.
\end{abstract}

Palabras clave: Industria; crecimiento industria; concentración industrial; problemas productivos estructurales

\begin{abstract}
The textile industry, which historically has been relevant for the Ecuadorian economy because of its influence in macroeconomics aggregates such the Gross Domestic Product (GDP) and the employment, has growth problems reflected in its production level. The objective of this article is to determine the principal cause of the low level of growth of this industry; for this reason, it will be checked the relationship between the industrial concentration and the level of growth of the industry. On the other hand, it will be determined the existence of entry barriers and productive problems that the Ecuadorian textile industry faces on, like causes of its low level of growth. In this article, it will be developed a qualitative and quantitative research. The principal results are that the textile industry presents a low level of industrial concentration and the entry barriers are not strong, for this reason, both do not have a direct influence in the low level of growth of the industry. Nevertheless, the Ecuadorian textile industry has structural problems derived from the industrial activities of the enterprises and the public policy. These problems are the principal cause of the growth problems of the industry.
\end{abstract}

Keywords: Industry; industrial growth; industrial concentration; structural productive problems

\section{Introducción}

Históricamente, la industria textil ecuatoriana se ha destacado por ser una de las actividades manufactureras con mayor relevancia económica para el país. Fue el pilar fundamental del desarrollo económico de la Real Audiencia de Quito (1563 - 1822), la misma que se constituyó como el principal centro de producción textil de las colonias españolas, basado en la fabricación de textiles y confecciones en la zona Centro Norte de la serranía ecuatoriana (Naranjo, 2009). Posteriormente, en la primera mitad del siglo XX, la industria textil ecuatoriana experimentó un importante periodo de crecimiento debido a factores internos y externos que permitieron un desarrollo incipiente de la producción textil, a través de empresas las cuales implementaron sistemas de producción tecnificados mediante la importación de maquinaria e implementación de energía eléctrica dentro del proceso productivo. Así, en aquella época, la industria textil llegó a ser el sector más industrializado del país (Cuvi, 2011).

Ya en el siglo XXI, con una economía ecuatoriana dolarizada desde el año 2000, se dinamizaron las exportaciones de productos textiles; sin embargo, medidas comerciales aperturistas implantadas en 2005 con países asiáticos permitieron el ingreso de textiles a menor precio, reduciendo la producción nacional. Para el año 2010, con un nuevo gobierno, se limitó la importación de textiles por medio de instrumentos comerciales como cuotas de importación y salvaguardias, permitiendo dar impulso a la industria nacional. Así, la industria textil ecuatoriana ha logrado posicionarse como la segunda industria manufacturera más importante del país (Arghoty, 2013).

Desde un enfoque cuantitativo, la relevancia económica de la industria textil ecuatoriana se la evalúa mediante el análisis de dos variables: el aporte económico al Producto Interno Bruto (PIB) y la generación de empleo. Según información estadística del Banco Central del Ecuador (2015), la industria textil ecuatoriana dentro del periodo 2000 - 2015 se constituye como la cuarta industria manufacturera con mayor aportación al PIB. A inicios del siglo XXI, específicamente en el año 2000, la industria textil tuvo una participación del 9\% y 2,01\% sobre el PIB manufacturero y PIB total, respectivamente. Sin embargo, para el año 2015, su participación se reduce, llegando a representar el 5,59\% y 0,80\% del PIB manufacturero y PIB total, respectivamente; por lo cual pasó a ser la quinta industria manufacturera con mayor participación en el PIB (BCE, 2015). (Véase Figura 1).

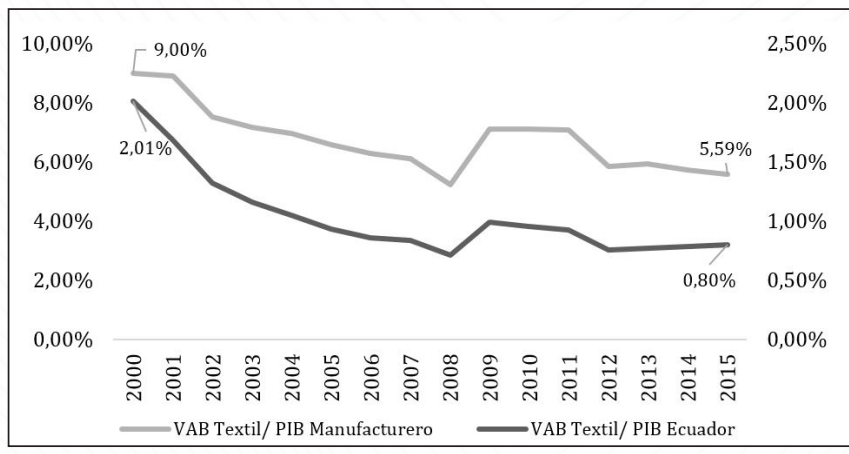

Fuente: Elaboración propia a partir de Banco Central del Ecuador, Cuentas Nacionales, VAB por industria (2015) Figura 1. Participación del VAB Textil en el PIB Ecuador y PIB manufacturero (Datos a valor corriente)

Respecto a la generación de empleo, la industria textil ecuatoriana se constituye como la segunda industria manufacturera que más empleo genera (BCE, 2015)3. En el año 2000, llegó a representar el 12,97\% del empleo total manufacturero. Sin embargo, para 2015, su nivel de participación sobre el empleo manufacturero se redujo al 7,11\%. Cabe destacar que el nivel de participación laboral de la industria textil, desde el año 2000 al 2015, siempre mantuvo tendencias a la baja, con escasas excepciones en tres años dentro del periodo de estudio (Véase Figura 2).

Artículo basado en la disertación de grado titulada "Industria textil ecuatoriana: relevancia económica, concentración industrial y barreras de entrada. Periodo 2000 - 2015", elaborada por Sebastián Amaluisa Peñaranda (2018).

2Pontificia Universidad Católica del Ecuador. Facultad de Economía. Quito - Ecuador. E-mail: andreesebas_1710@hotmail.com. ORCID: https://orcid.org/0000-0002-3472-1805

${ }^{3}$ La primera industria manufacturera en generación de empleo es la industria de elaboración de alimentos y bebidas (44,88\% de participación promedio en el periodo 2000 - 2015) 


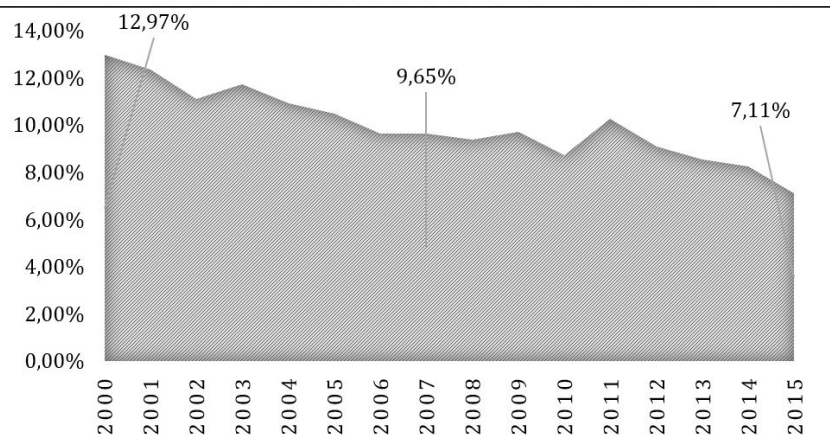

Fuente: Elaboración propia a partir de Instituto Nacional de Estadísticas y Censos, Encuesta de Manufactura y Minería, Personal ocupado (2015)

Figura 2. Participación de la industria textil en generación de empleo manufacturero

A pesar de la importancia económica que genera la industria, se puede observar mediante los datos expuestos que, a través del tiempo, su nivel de representatividad sobre las dos variables macroeconómicas se reduce sustancialmente, lo cual es un indicador de que la industria se ha ido contrayendo y presentando problemas estructurales, especialmente de crecimiento. En este sentido, para determinar el nivel de crecimiento de la industria textil, se analiza la variable producción durante el periodo 2000 - 2015. Se pueden observar amplios periodos de volatilidad, en años donde el nivel de producción de la industria crece, pero al año siguiente decrece. Esto se debe sustancialmente a incrementos y decrementos de la importación de productos textiles y prendas de vestir, regulada por el establecimiento de salvaguardias por balanza de pagos (Véase Figura 3).

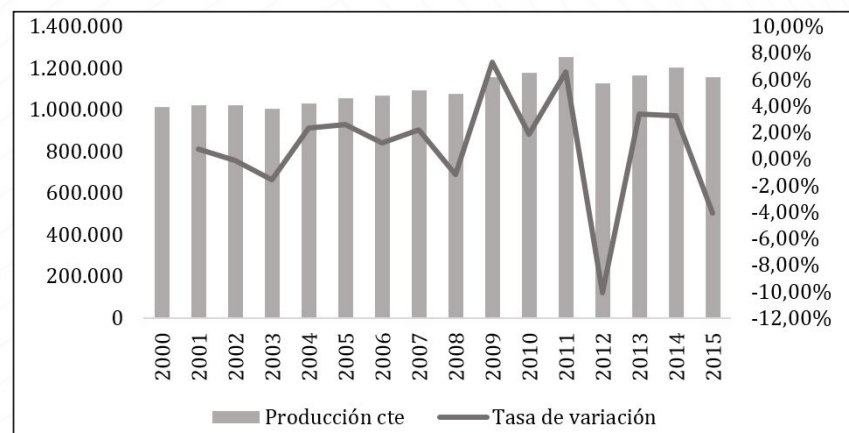

Fuente: Elaboración propia a partir del Banco Central del Ecuador, Cuentas Nacionales, Producción de las Industrias Figura 3. Producción de la industria textil ecuatoriana* y tasas de variación (Datos a valor constante en miles USD 2007)

Como consecuencia de la alta volatilidad del nivel de producción textil, durante el periodo 2000 - 2015, su nivel de producción presenta una tasa de crecimiento promedio del 0,95\% en 15 años. Esto indica claramente que la industria textil ecuatoriana presenta problemas de crecimiento. En conclusión, a pesar de ser económicamente relevante, la industria textil ecuatoriana crece lentamente.

Con el fin de determinar la causa del bajo nivel de crecimiento de la industria reflejado en su volátil nivel de producción, se tomarán como base dos postulados. El primero establece que el nivel de crecimiento de una industria tiene una relación inversa con cambios en el nivel de concentración industrial; es decir, el bajo nivel de crecimiento de la industria textil ecuatoriana puede tener su causa en un alto nivel de concentración industrial de las empresas que conforman el sector (Curry y George, 1983).

Por otro lado, Porter (1985) en su libro "Ser Competitivo", establece varias determinantes que puede influir, o no, en la competitividad y crecimiento de un sector económico. Una de las determinantes son las barreras de entrada, las mismas que con su existencia pueden conllevar a la generación de concentración industrial. Sin embargo, las barre- ras de entrada se originan a partir de problemas derivados del accionar productivo de las empresas y de la política gubernamental. Éstos dos últimos factores serán el punto de análisis para establecer si existen problemas estructurales que impiden un crecimiento favorable para la industria textil ecuatoriana.

Por tal motivo, la presente investigación tiene como objetivo determinar cuál es la causa fundamental del bajo nivel de crecimiento de la industria textil ecuatoriana: un alto nivel de concentración industrial o problemas productivos estructurales.

\section{Metodología}

La presente investigación es de tipo descriptiva, debido a que establece la observación y posterior descripción de datos y variables respecto a la industria textil ecuatoriana. Además, el tipo de análisis de la investigación es de carácter mixto, en el sentido en que abarca un enfoque cuantitativo y cualitativo. Respecto al análisis cuantitativo, en la investigación se desarrolla un análisis de concentración de las empresas que conforman la industria textil ecuatoriana con el objetivo de comprobar el cumplimiento del postulado propuesto respecto a la relación inversa existente entre crecimiento industrial y concentración industrial.

Para esto, se aplicarán dos herramientas estadísticas: el cálculo de índice de concentración de Herfindahl-Hirschman (HHI) y con los resultados obtenidos mediante el cálculo del HHI, posteriormente se procede a realizar el cálculo del coeficiente de correlación entre las variables crecimiento industrial (VAB textil) y concentración industrial $(\mathrm{HHI})$. Por otro lado, se procede a realizar un análisis cualitativo del sector mediante entrevistas a dos especialistas textiles: al presidente de la Asociación de Industriales Textiles del Ecuador, Ing. Javier Díaz Crespo; y al presidente de la Asociación de Confeccionistas Textiles, Dr. Milton Altamirano. Estas entrevistas tienen el fin de buscar información primaria que permitan conocer la realidad en la cual se desarrolla la industria textil ecuatoriana y establecer si existen problemas productivos de tipo estructural que afecten al crecimiento de la industria.

Respecto a los datos a utilizarse en el desarrollo de la presente investigación, se toma como base la información de fuentes oficiales nacionales. A partir de las Cuentas nacionales del Banco Central de Ecuador (BCE), se extrae información respecto a la incidencia de la industria sobre el PIB a partir de los datos del Valor Agregado Bruto (VAB). Además, los datos de la producción industrial también son extraídos de las cuentas nacionales del BCE. Por su parte, la información respecto a la generación de empleo industrial tiene como fuente de datos la Encuesta de Manufactura y Minería del Instituto Ecuatoriano de Estadística y Censos (INEC).

Para la realización de los cálculos del índice de concentración de Herfindahl-Hirschman, se utiliza la variable Ingreso total, concerniente a todas las empresas de la industria textil (subsector C13 y C14) correspondiente a los Balances de compañías manufactureras, presentes en el portal de información de la Superintendencia de Compañías, Valores y Seguros.

\section{Resultados}

Para establecer cuál de los dos planteamientos señalados anteriormente genera influencia sobre el bajo nivel de crecimiento de la industria textil ecuatoriana, se proceden a realizar los siguientes análisis:

\section{Análisis de concentración de la industria textil ecuatoriana}

Para determinar el nivel de concentración industrial de la industria textil ecuatoriana, se procede a calcular el índice de Herfindahl-Hirschman ${ }^{4}$ para las empresas pertenecientes a la industria textil, la cual, según el Código Internacional Industrial Uniforme (CIIU), se compone de dos subsectores: fabricación de productos textiles (CIIU C13) y fabricación de prendas de vestir (CIIU C14). Las empresas a analizar son aquellas

${ }^{4}$ El índice de Herfindahl se expresa: $H H I=\sum_{i=1}^{N} s_{i}^{2} \rightarrow H H I=\sum_{i=1}^{N}\left(\frac{x_{i}}{x} \times 100\right)^{2} ;$ Donde: $\frac{x_{i}}{x}=$ Participación porcentual de la i-ésima empresa en el mercado; $N=$ Número de firmas participantes. Es decir, el índice Herfindahl es la suma del cuadrado de las porciones individuales de mercado de todas las empresas incluidas en el sector industrial de análisis (Gutiérrez y Zamudio, 2008)

Bajo nivel de crecimiento de la industria textil ecuatoriana: ¿Elevada concentración industrial o problemas productivos estructurales? 
que pertenecen a los subsectores $\mathrm{C} 13$ y $\mathrm{C} 14$, y que se encuentran registradas en la Superintendencia de Compañías, Valores y Seguros, entre los años 2007 a 2015.

Para el cálculo de concentración industrial, la variable de estudio de las empresas es su ingreso total. A partir de esta variable, se determinará la cuota de mercado de cada empresa, la cual a su vez es una componente para el cálculo del índice de concentración. En la figura 4, se puede observar los resultados obtenidos a partir del cálculo del índice de Herfindahl-Hirschman, para las empresas perteneciente a la industria textil ecuatoriana (Subsector de fabricación de productos textiles C13 y fabricación de prendas de vestir C14).

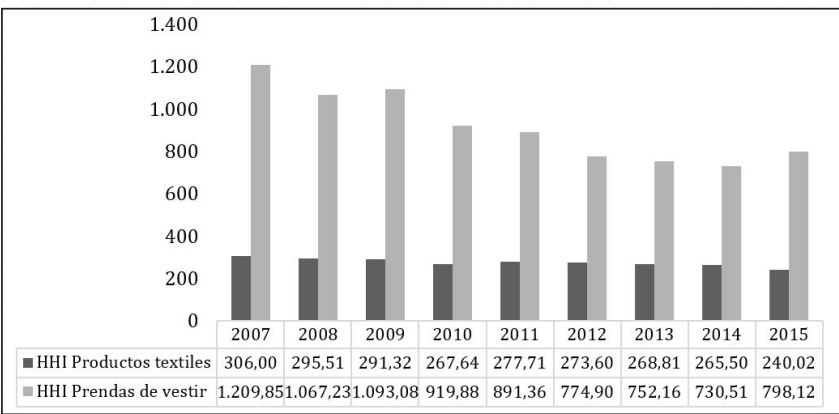

Fuente: Elaboración propia a partir de la Superintendencia de Compañías, Valores y Seguros, Balances de Compañías Manufactureras Figura 4. Índice de Herfindahl Hirschman por subsector textil

Basado en los resultados obtenidos mediante el cálculo del índice de concentración de Herfindahl-Hirschman y en los parámetros de interpretación $n^{5}$ del índice, se puede determinar que el nivel de concentración industrial para la industria textil en el periodo 2007 - 2015 es bajo.

El subsector de fabricación de productos textiles (C13) mantiene un índice de Herfindahl-Hirschman que oscila entre los 306,00 puntos hasta los 240,02 puntos; mientras que el subsector de fabricación de prendas de vestir presenta un índice de Herfindahl-Hirschman que va entre los 1.209,85 puntos a 730,51 puntos, en el periodo $2007-2015$. Cabe destacar que, año a año dentro del periodo de estudio, el índice de Herfindahl-Hirschman disminuye progresivamente en ambos subsectores textiles, reflejando el bajo nivel de concentración de la industria (Solano, Camino y Alvarado, 2017).

Al haber calculado el índice de concentración industrial, se procede a determinar la relación existente entre el nivel de crecimiento de la industria textil ecuatoriana y su nivel de concentración industrial. Por tal motivo, se realiza el cálculo del coeficiente de correlación entre estas las dos variables: el nivel de crecimiento determinado por el VAB de los subsectores C13 y C14; y el nivel de concentración industrial determinado por los valores obtenidos mediante el cálculo del índice de Herfindahl-Hirschman; dentro del periodo 2007 - 2015.

\begin{tabular}{lcccc}
\multicolumn{5}{c}{ Tabla 1. Nivel de crecimiento $y$ de concentración por subsector textil } \\
\hline $\begin{array}{c}\text { VAB C13 MILES } \\
\text { USD }\end{array}$ & HHI C13 & VAB C14 MILES USD & HHI C13 \\
$\mathbf{2 0 0 7}$ & 193.129 & 306,00 & 235.241 & $1.209,85$ \\
$\mathbf{2 0 0 8}$ & 209.745 & 295,51 & 232.493 & $1.067,23$ \\
$\mathbf{2 0 0 9}$ & 309.534 & 291,32 & 308.887 & $1.093,08$ \\
$\mathbf{2 0 1 0}$ & 345.704 & 267,64 & 319.115 & 919,88 \\
$\mathbf{2 0 1 1}$ & 342.386 & 277,71 & 389.192 & 891,36 \\
$\mathbf{2 0 1 2}$ & 268.415 & 273,60 & 396.867 & 774,9 \\
$\mathbf{2 0 1 3}$ & 327.992 & 268,81 & 408.879 & 752,16 \\
$\mathbf{2 0 1 4}$ & 349.798 & 265,50 & 446.773 & 730,51 \\
$\mathbf{2 0 1 5}$ & 367.941 & 240,02 & 429.243 & 798,12 \\
\hline
\end{tabular}

Fuente: Elaboración propia a partir de Banco Central del Ecuador, Cuentas Nacionales, VAB por industria (2015) y de los resultados obtenidos a partir del cálculo del índice de Herfindahl - Hirschman.

${ }^{5}$ El índice de Herfindahl-Hirschman (HHI) toma valores dentro del rango cero y diez mil (0 $\leq$ HHI $\left.\leq 10000\right)$, y se lo interpreta de la siguiente forma: Bajo nivel de concentración $(0 \leq H H I \leq 1,000)$, Moderado nivel de concentración (1,000 $\leq H H I \leq 1,800)$, Alto nivel de concentración (1,800 $\leq H H I)$. (Gutiérrez y Zamudio, 2008) Bajo nivel de crecimiento de la industria textil ecuatoriana: ¿Elevada concentración industrial o problemas productivos estructurales?
En la Tabla 2, se pueden observar los resultados obtenidos mediante el cálculo del coeficiente de correlación entre el nivel de crecimiento y el nivel de concentración de la industria textil ecuatoriana.

Tabla 2. Resultados del cálculo del coeficiente de correlación entre crecimiento y concentración industrial de la industria textil ecuatoriana

\begin{tabular}{|c|c|c|c|c|c|}
\hline & $V A B C 13$ & HHI & & VAB C14 & ннI \\
\hline VAB C13 & 1 & & VAB C14 & 1 & \\
\hline нНI & $-0,8276087$ & 1 & HHI & $-0,928998$ & 1 \\
\hline
\end{tabular}

Fuente: Elaboración propia a partir de los resultados obtenidos a partir del cálculo del índice de Herfindahl - Hirschman

A partir de los resultados obtenidos, se puede determinar que existe una correlación negativa entre la variable del valor agregado bruto (VAB) de los subsectores pertenecientes a la industria textil ecuatoriana (C13 y C14) con la variable de concentración industrial representada por los valores obtenidos del cálculo del índice de Herfindahl-Hirschman: correlación de -0,83 para el subsector C13 y - 0,93 para el subsector C14; entre ambas variables.

En conclusión, se establece que el nivel de concentración industrial no presenta influencia sobre el nivel de crecimiento de la industria textil ecuatoriana en el periodo 2007 - 2015. Esto se explica debido a que, al ser una industria no concentrada, no existen empresas que ejerzan poder de mercado e impidan el desarrollo del resto de empresas pertenecientes a la industria. Por lo tanto, esta no es la causa de un bajo nivel de crecimiento de la producción de los subsectores de fabricación de productos textiles (C13) ni de fabricación de prendas de vestir (C14).

Por este motivo, el planteamiento teórico realizado por Curry y George (1983) respecto a la relación inversa entre crecimiento y concentración industrial, no se cumple para el caso de la industria textil ecuatoriana y no explica el bajo nivel de crecimiento de esta industria.

Debido a que la industria textil ecuatoriana no presenta un alto nivel de concentración industria, y esta condición tampoco explica el bajo nivel de crecimiento de la industria; a continuación, se procede a realizar un análisis cualitativo de la situación por la cuál atraviesa la industria textil, con el fin de determinar la existencia de barreras de entrada y problemas estructurales de tipo productivo que enfrente este sector manufacturero.

Análisis de barreras de entrada y problemas productivos en la industria textil ecuatoriana

Con el fin de obtener información detallada respecto a la situación que enfrenta la industria textil, en primera instancia, se procede a realizar entrevistas a dos especialistas del sector: al presidente de la Asociación de Industriales Textiles de Ecuador (AITE), el Ingeniero Javier Díaz Crespo; y al presidente de la Asociación de Confeccionistas Textiles (ACONTEX), el Doctor Milton Altamirano.

A partir de la información obtenida mediante las entrevistas a los especialistas textiles, a continuación, se procede a realizar un análisis de la existencia de barreras de entrada en la industria textil ecuatoriana en base a la información previamente expuesta. 


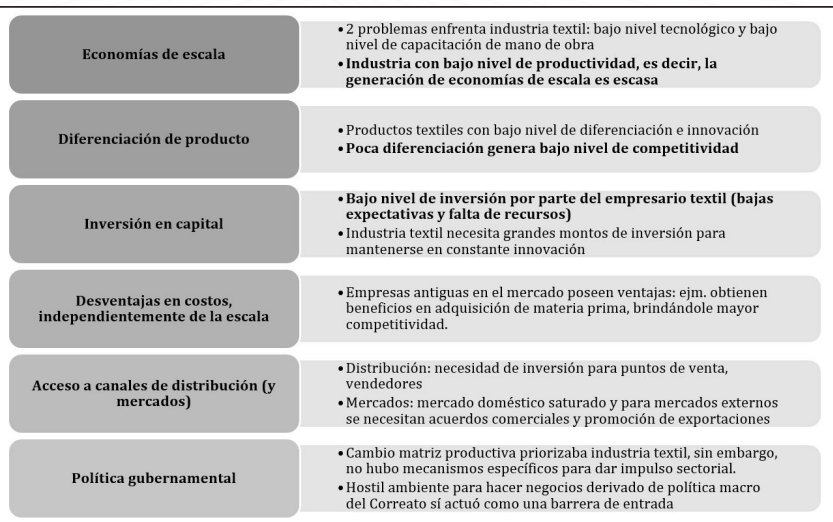

Fuente: Elaboración propia a partir de la información obtenida a partir de entrevistas realizadas a especialistas textiles

Figura 5. Análisis de la existencia de barreras de entrada en la industria textil ecuatoriana

Como se puede observar en la Figura 5, no se puede definir la presencia real de barreras de entrada en la industria textil ecuatoriana. Por tal motivo, la no existencia de barreras de entrada tampoco explicaría el bajo nivel de crecimiento de la industria textil ecuatoriana. Por el contrario, la dificultad para generar economías de escala, el bajo nivel de diferenciación de productos y el escaso acceso a canales de distribución y mercados son problemas que no permiten que la industria textil crezca, restándole productividad y competitividad.

En conclusión, se establece que las barreras de entrada, per sé, no generarían influencia directa sobre el nivel de crecimiento de la industria textil ecuatoriana en el periodo 2007 - 2015. Sin embargo, sí existen dificultades industriales que no permiten el crecimiento sostenido de la industria textil ecuatoriana.

A estas dificultades que enfrenta la industria textil ecuatoriana se las puede catalogar como problemas productivos estructurales, los mismos que se originan por dificultades propias de la industria (problemas micro) y problemas derivados por el accionar de la política gubernamental (problemas macro). Cabe destacar que los problemas estructurales presentes en la industria sí afectan a su nivel de crecimiento, debido a que no permiten alcanzar un alto grado de productividad y competitividad en el sector textil ecuatoriano.

\section{Conclusiones}

A pesar de la importancia histórica y económica que representa la industria textil ecuatoriana, misma que se refleja a través de su nivel de aportación al PIB manufacturero, siendo la quinta mayor industria con el 5,59\% de aporte en el año 2015; y en cuanto a la generación de empleo, siendo la segunda mayor industria generadora de empleo con el 7,11\% en el año 2015; la industria textil ecuatoriana presenta un bajo nivel de crecimiento durante el periodo 2000 - 2015, reflejado en su nivel de producción. La variable producción indica que, durante los 15 años de estudio, la industria textil alcanza una tasa de crecimiento del $0,95 \%$, la cual es baja para una de las industrias más relevantes para el país. Esto se debe principalmente a un volátil nivel de producción interanual.

A través del análisis de concentración industrial, mediante el cálculo del índice de Herfindahl-Hirschman, se establece que la concentración industrial es baja dentro de la industria textil ecuatoriana. Los valores obtenidos fluctúan entre los 240,02 puntos y 1209,85 puntos, lo cual expresan un bajo nivel de concentración industrial. A partir del cálculo del coeficiente de correlación entre el nivel de crecimiento de la industria, expresada por el VAB sectorial, y el nivel de concentración, expresada mediante los índices obtenidos mediante el cálculo del índice de Herfindahl-Hirschman, se establece que existe una correlación negativa entre estas dos variables. Es así que la teoría económica que explica una relación negativa entre el nivel de crecimiento de la industria y su nivel de concentración, propuesta por Curry y George (1983), no se cumpliría. En conclusión, la concentración industrial no explica el bajo nivel de crecimiento de la industria textil ecuatoriana.

Al analizar la existencia de barreras de entrada en la industria textil ecuatoriana, se puede establecer en primera instancia, la no existencia de verdaderas barreras de entrada debido a las dificultades que enfrenta la industria textil para generarlas. Por lo tanto, éstas tampoco explicarían el bajo nivel de crecimiento de la industria. No obstante, mediante la información y datos obtenidos a partir de las entrevistas con los dos especialistas textiles, se puede destacar que la industria textil ecuatoriana presenta problemas estructurales que no permiten un crecimiento sostenido de su producción.

Por lo tanto, la principal y verdadera causa del bajo nivel de crecimiento de la industria son problemas productivos estructurales de dos tipos: propios de la industria y derivados de la política aplicada por el gobierno nacional. Estos generan a su vez problemas subyacentes en la industria: bajo nivel de productividad y competitividad industrial; impidiendo su crecimiento en el tiempo.

Al haber realizado la comprobación empírica de las teorías planteadas por Curry y George (1983) y Porter (1985), se puede establecer que, tanto la concentración industrial como la presencia de barreras de entrada, no explican el caso del bajo nivel de crecimiento de la industria textil ecuatoriana tomando en consideración el estudio de la variable producción. Se considera el análisis y posterior comprobación de ambos postulados teóricos debido a que son considerados como uno de los determinantes del nivel de crecimiento de una industria. Sin embargo, mediante los resultados obtenidos, se establece que la industria textil posee bajo nivel de concentración industrial y que no presenta barreras de entrada relevantes que puedan afectar al desarrollo de la industria.

\section{Referencias}

Amaluisa, Sebastián (2018) Industria textil ecuatoriana: relevancia económica, concentración industrial y barreras de entrada. Periodo 2000 - 2015 (Disertación de Economía). Recuperada de Repositorio digital de la PUCE: http:// repositorio.puce.edu.ec/handle/22000/15619

Banco Central del Ecuador (2015) Cuentas nacionales anuales

Curry, B y George, K (1983) Industrial Concentration: A Survey. Journal of Industrial Economics, 31

Cuvi, Nicolás (2011) Auge y decadencia de la Fabrica de Hilados y Tejidos de algodón "La Industrial". Revista Procesos, 33 (65-73). Recuperado de: http://repositorio. uasb.edu.ec/bitstream/10644/3046/1/04-ES-Cuvi-s.pdf

Gutiérrez, Javier y Zamudio, Nancy (2008) Medidas de Concentración y competencia. Departamento de estabilidad Financiera. Bogotá: Banco de la República

Instituto Nacional de Estadísticas y Censos (2012) Clasificación Nacional de Actividades Económicas. Unidad de Análisis y sintesis.

Naranjo Chiriboga, Marco (2009) Formación Socioeconómica del ECuador, Diplomacia Etrategia Política, Fundación Alexander de Gusmao, Brasilia

Porter, Michael (1985) Ser Competitivo. Bogotá: Paidós.

Solano, Javier; Camino, Segundo y Alvarado María (2017) Análisis del entorno competitivo en el que operan las Mypimes del sector manufacturero en Ecuador. Revista Empresarial, 11(44), 53- 62. Guayaquil: UCSG.

Superintendencia de Compañías, Valores y Seguros (2015) Portal de Información - Sector Societario - Información estadística - Balances de Compañías Manufactureras. 